\title{
Cognitive Decline Associated with Normal Aging in Rats: A Neuropsychological Approach
}

\author{
David R. Zyzak, Tim Otto, ${ }^{1}$ Howard Eichenbaum, ${ }^{2,3}$ and Michela Gallagher \\ Department of Psychology \\ University of North Carolina at Chapel Hill \\ Chapel Hill, North Carolina 27599-3270
}

\section{Abstract}

The effects of aging on cognitive capacities were examined by comparing the performance of young and old rats on tasks characterized as dependent on different brain systems. This neuropsychological approach was employed to determine the extent to which multiple neural systems are compromised in aging and whether deterioration of one system correlates with that of another. The two tasks used in the present study were an odor-guided recognition memory task, for which different aspects of performance have been shown to be dependent on the integrity of the orbital prefrontal and perirhinal-entorhinal cortex, and the Morris water maze, for which performance depends on the medial prefrontal cortex and hippocampus. Rats were trained on the recognition memory task under minimal memory load and then challenged with longer memory delays and higher levels of inter-item interference. Considerable variation was observed in the performance of aged rats on acquisition of the recognition memory task, and unlike young rats, some aged rats could not acquire the task. Nevertheless, those aged rats who did acquire the cDNM task performed as well as young animals when the memory delay was extended and interference was elevated. In

Present addresses: ${ }^{1}$ Department of Psychology, Rutgers University, Busch Campus, New Brunswick, New Jersey 08903; ${ }^{2}$ Center for Behavioral Neuroscience, State University of New York at Stony Brook, Stony Brook, New York 11794-2575.

${ }^{3}$ Corresponding author. addition, consistent with previous findings, the performance of the same aged rats was highly variable in the Morris water maze task.' Furthermore, although correlations between scores on the two tasks for individual aged rats were not reliable, only those aged rats that performed outside the performance range of young rats in the water maze were impaired on acquisition of the recognition memory task. This pattern of findings is consistent with age-related dysfunction in multiple subdivisions of the prefrontal cortex as well as the hippocampus and suggests that these brain regions may deteriorate in the same subgroup of aged rats.

\section{Introduction}

The neuropsychological study of aging in humans attempts to relate changes in cognitive function in elderly adults to alterations in neural systems. Researchers using computerized tomography (CT), positron emission tomography (PET), and magnetic resonance imaging (MRI) scans have concentrated on identifying changes in the aged brain that significantly differentiate patients with Alzheimer's disease and other age-related dementias from aged controls (de Leon et al. 1992; Sandor et al. 1992). In addition to demonstrating the locus of brain atrophy in Alzheimer's disease, research using brain imaging techniques has also revealed hippocampal atrophy in nondemented aged subjects, indicating that those aged subjects experiencing hippocampal atrophy perform more poorly on tests of verbal memory compared with aged subjects without hippocampal atrophy (Golomb et al. 1993, 1994). This neuroanatomical basis for age-related memory decline is congruent

LEARNING \& MEMORY 2:1-16 (c) 1995 by Cold Spring Harbor Laboratory Press ISSN1072-0502/95 \$5.00

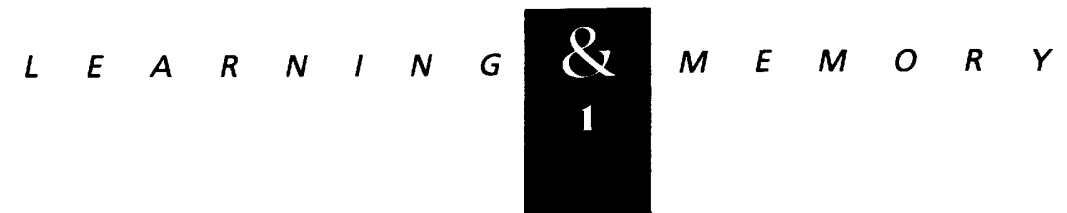


with much experimental research indicating that medial temporal lobe systems are critical for normal memory function.

Other studies have revealed that the prefrontal cortex is also compromised in aging and that some aspects of behavioral and cognitive decline in aging parallel the effects of frank damage to the prefrontal region (for reviews, see Rapp and Heindel 1994; Shimamura 1994). The combination of these studies implicates multiple brain systems in the profile of cognitive deterioration in normal aging in humans. In addition, many of these studies indicate considerable variability in both the locus and extent of brain alterations and cognitive impairment associated with aging (Shimamura 1994).

Animal models of aging would be highly useful in investigations of the cellular and molecular bases of age-related cognitive decline. Recent studies on rats and monkeys have revealed deficits in cognitive capacities associated with aging and have shown that, as in humans, the profile of cognitive deficits is broad (Dunnett et al. 1988; Winocur and Moscovitch 1990; Rapp and Amaral $1989,1992)$ and that the severity of the cognitive decline associated with aging is highly variable in animals (Collier and Coleman 1991; Rapp and Amaral 1991, 1992; Gallagher et al. 1995). Furthermore, considerable recent research has successfully identified some of the brain systems critical to performance in different cognitive tasks used to study cognitive capacities in animals. In this study a neuropsychological approach was employed to examine the effects of aging on the cognitive capacities of rats. The performance of young and aged rats was compared on two different learning and memory tasks that are dependent on distinct brain systems.

One task that was employed was an odorguided continuous delayed nonmatch-to-sample task (cDNM) (Otto and Eichenbaum 1992) analogous to the visually guided delayed nonmatch-tosample task used so successfully in studies of medial temporal lobe function in primates (Gaffan 1974; Mishkin 1982; Zola-Morgan and Squire 1985). In this task, rats are required to recognize recently experienced odors. They are presented with a succession of stimulus items and must judge each one as the same as (matching) or different from (nonmatching) the preceding item. The time interval between presentations is altered to assess the capacity for retention over a delay, and the size of the pool of stimulus items is varied to assess performance under different levels of inter-item interference. The neuropsychological assessment of cDNM performance in rats provides some important parallels with nonhuman primate research. As in both human recognition memory and performance on delayed nonmatch to sample in nonhuman primates, performance by rats on the cDNM task is sensitive to parametric manipulations that increase memory demands (increasing the memory delay or interference level). Moreover, cDNM performance in the rat is sensitive to hippocampal system lesions that also produce impairments on visual and tactile cued delayed nonmatch to sample in primates (Zola-Morgan et al. 1989; Meunier et al. 1993; Suzuki et al. 1993). In the rat, perirhinal and entorhinal cortical lesions do not affect cDNM acquisition or short-delay retention but result in progressively greater impairments in performance at longer delays and with higher levels of interference (Otto and Eichenbaum 1992). In contrast, selective neurotoxic lesions of the hippocampus itself or transection of the fornix produce neither acquisition nor delaydependent deficits in CDNM rat performance (Otto and Eichenbaum 1992; Wilner et al. 1993). Similarly, perirhinal-parahippocampal and entorhinal cortex lesions produce profound delaydependent deficits on delayed nonmatch to sample in nonhuman primates but selective neurotoxic or stereotaxic lesions of the hippocampus proper result in very little if any impairment under similar testing conditions (Zola-Morgan et al. 1989; O'Boyle et al. 1993). In nonhuman primates, aging results in impairments in both initial acquisition of delayed nonmatch-to-sample task and a selective impairment of delayed recognition in postacquisition performance in a subgroup of subjects (Rapp and Amaral 1991). One main aim of the present research was to assess the effects of aging on recognition memory in rats as reflected in acquisition and delay performance on the cDNM task.

The other paradigm employed in the present study was the Morris water maze task. The Morris water maze task has been widely used to assess hippocampal/temporal lobe function in spatial learning capacities of rats (Barnes 1979; Mitchell et al. 1982; Morris et al. 1982, 1986, 1990). In aged rats there are striking individual differences in performance, such that some aged rats learn the task as proficiently as young rats, whereas a subset of the aged rats perform similarly to young rats with hippocampal system damage (Gallagher and

$$
\begin{array}{llllllll|c|cccccc} 
& E & A & R & N & I & N & G & \begin{array}{l}
\boldsymbol{X} \\
2
\end{array} & M & E & M & O & R & Y
\end{array}
$$


Pellymounter 1988; Gallagher and Burwell 1989; Gallagher et al. 1993). Additional studies have provided support for the hypothesis that hippocampal system dysfunction underlies the cognitive decline in spatial learning ability during normal aging (Barnes 1979; for review, see Gallagher et al. 1995). The other main aim of the present research was to assess the extent to which age-related decline in spatial learning performance reflected a general cognitive impairment that would extend to recognition memory.

Determining the neurobiological basis for agerelated loss of spatial learning ability may provide a suitable rodent model for the study of aging in the rodent hippocampal system. However, it is uncertain whether the neural substrate for this cognitive ability in the medial temporal lobe is similar to that on which recognition memory in the cDNM task depends. Whereas intact performance in the cDNM task can be achieved by rats with damage to the hippocampus proper, such lesions impair spatial learning in young rats. Perirhinalentorhinal damage, on the other hand, produces both delay-dependent CDNM deficits and impairment in spatial learning in rats (Otto and Eichenbaum 1992; Nagahara et al. 1995). In addition, damage to the distinct subdivisions of the prefrontal cortex results in selective impairments in the acquisition of olfactory and spatial memory tasks (Eichenbaum et al. 1983; Otto and Eichenbaum 1992). Thus, comparing these characterizations of olfactory and spatial learning with particular profiles of deficient and intact abilities in aged rats might indicate which brain areas are compromised in aging. The differing lesion profiles for recognition memory and spatial learning could also be brought to bear on determining whether a common neurobiological substrate contributes to the broad range of age-related cognitive impairments. If this were the case, not only would impairment be evident in each task, but a consideration of individual differences might reveal a correlation in the severity of impairment in aged animals in the same study population assessed in both tasks.

The following experiment was designed to use neuropsychological techniques in the study of cognitive decline in aged rats by examining the extent to which performance across spatial and cDNM tasks was selectively or concurrently affected. The same groups of young and aged rats were tested in the odor CDNM maze using varying conditions of delay and interference and in both the place and cue versions of Morris water maze.
We were particularly interested in whether there is an age-related impairment in simple recognition memory in rats and, upon identifying such an impairment, whether the severity of the recognition memory deficit would correlate with the spatial learning impairment demonstrated previously in the same strain of aged rats.

\section{Materials and Methods}

\section{SUBJECTS}

Forty-three male (17 young, 6 months, 26 aged, 25 months at the outset of testing), pathogen-free, Long-Evans rats were used. The aged rats were obtained as retired breeders at 9 months of age and young rats were obtained at 90 days of age (Charles River Labs, Raleigh, NC). Subjects were maintained on a 12:12-hr light-dark cycle (lights on at 7A.M.) in an environmentally controlled room. All behavioral training was conducted during the light cycle. Food was available ad libitum throughout the study. Water access was restricted to a 30-min period at the end of each day during the odor memory test. Routine screening for viral antibodies indicated that the aged rats were healthy at the end of the study. Necropsies performed at sacrifice revealed pituitary tumors in two aged rats that were eliminated from the study on that basis.

\section{APPARATUS}

Behavioral testing in cDNM took place in a $30-\mathrm{cm}$ square Plexiglas chamber that was enclosed in a sound-attenuating Plexiglas box. A conical sniff port was centered on one wall of the chamber $5 \mathrm{~cm}$ above the floor. A water port $(1-\mathrm{cm}$ diam $\times 1$ $\mathrm{cm}$ depth) was located directly above the sniff port. Responses at the sniff and water ports were monitored by separate infrared photoelectric beams. Two $24 \mathrm{~V}$ light bulbs (referred to as "house lights") were mounted (13 cm above floor) on either side immediately above the sniff and water ports. Individual odors were delivered as required by a 16-channel flow-dilution olfactometer. Clean air flowed continuously at a rate of $0.5 \mathrm{liter} / \mathrm{min}$ with odorized airstreams at the same delivery rate added to the clean air when required so as to deliver a final flow rate of $1.0 \mathrm{liter} / \mathrm{min}$. Presentation of odorized and clean airstreams was controlled by

$$
\begin{array}{llllllll|c|cccccc}
L & E & A & R & N & I & N & G & \mathcal{Q} & M & E & M & O & R & Y \\
3 & & & & & &
\end{array}
$$


a solenoid valve mounted immediately outside the behavioral chamber; during the delay period (i.e., intertrial interval), the airstreams were diverted by the valve to a vacuum dump flowing at twice the final odor flow rate. Odors were presented to the subject by shutting off the vacuum solenoid, thereby allowing the odorized airstream to enter the behavioral chamber through the sniff port. A fan mounted on the outside of the sound-attenuating chamber provided continuous air turnover, removing any lingering odors. All procedural events were controlled and behavioral responses recorded by a microcomputer with custom-designed interfaces.

Spatial learning was assessed in a Morris water maze apparatus. The water maze consisted of a circular tank (diam., $1.83 \mathrm{~m}$; height, $0.58 \mathrm{~m}$ ) filled with tepid water $\left(27^{\circ} \mathrm{C}\right)$ opacified by powdered milk. A white escape platform (height, $34.5 \mathrm{~cm}$ ) was located $1 \mathrm{~cm}$ below the water surface near the center of one of the four quadrants of the maze for the spatial learning task. A visible black escape platform was used for cue training. The maze was surrounded by white curtains with patterns affixed to provide a configuration of spatial cues. Data were analyzed by a video tracking system (HVS Imaging, VP112) and microcomputer with custom designed software (Richard Baker, HVS Imaging) developed specifically for this task.

\section{PROCEDURE: ODOR-GUIDED CONTINUOUS DELAYED NONMATCH TO SAMPLE}

Training and testing on the CDNM task was carried out in three phases. Each of these phases is described below.

SHAPING

Each water-deprived rat was shaped to place its nose into the sniff port and to retrieve water reinforcers at the water port. Subsequently, the onset of the house lights signaled the rat that it could initiate the trial with a nose poke onto the sniff port. A 500-msec nose poke resulted in the presentation of a single odor selected pseudorandomly from a set of 16 odors. During shaping sessions, the odor presented on each trial was always different from that presented on the immediately preceding trial. Water port responses terminated odor presentation and were reinforced with 0.05 ml of water. During each 3-sec intertrial interval the house lights were extinguished; nose pokes into the sniff port during the last 2 sec of the delay extended the delay by an additional $2 \mathrm{sec}$. Rats were required to successfully complete two successive 60 trial shaping sessions before beginning cDNM training procedures.

\section{TRAINING TO CRITERION}

Nonmatching training procedures differed from initial shaping sessions in the sequence of odors presented, the reward contingencies, the number of trials per session, and in the length of the delay. During training, unlike shaping, the odor presented on half of the trials differed from that presented on the preceding trial; on the other half of the trials, the odor was the same as that presented on the preceding trial. Responses to the water port during training were reinforced only if the odor on the current trial was different from that presented on the preceding trial. Thus, the rat was required to remember across the delay the odor presented on the immediately previous trial, and to respond by entering the water port only if the current odor was a "nonmatch." Water port responses on "match" trials were not reinforced and resulted in the house lights being extinguished. If no water port response was made within 5 sec of the odor onset, the odor and the house lights were turned off simultaneously, thus starting the delay. Correct responses were followed by a 3-sec delay; incorrect responses were followed by a 7-sec delay. Thus, correct responses were "go" responses on nonmatch trials and "nogo" responses on match trials. Incorrect responses were either errors of commission, "go" responses on match trials, or errors of omission, "no-go" responses on nonmatch trials. Two 50-trial sessions, one in the morning and one in the afternoon, were run daily 6 days/week.

A correction procedure for errors of commission was used early in training because previous studies had found that repetitions of these trials improves cDNM acquisition rate in young animals (Otto and Eichenbaum 1992). Correction trials had the same response contingencies as the match trial on which the error of commission was made. At most, two correction trials were presented, followed immediately by the presentation of the random odor sequence for the remaining session. Correction procedures were discontinued when

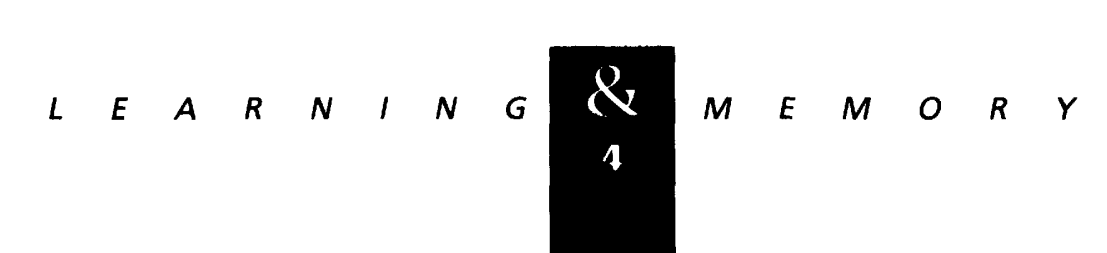


the rat reached a performance criterion of 18 correct responses in 20 consecutive trials. Correction trials were not calculated in the number of trials to reach criterion. Upon establishing this initial criterion, an additional criterion was required where subjects were trained in 50-trial sessions using a 3 -sec delay following every trial regardless of the response until task performance was $>\mathbf{8 5} \%$ correct on each of two consecutive training sessions. Training was continued for up to 600 trials to reach the first acquisition criterion and 1300 trials to reach the second. Some aged subjects did not reach one of these criteria and were subsequently discontinued in CDNM testing.

TESTING WITH INCREASED MEMORY DELAY AND INTER-ITEM INTERFERENCE

Following acquisition of the cDNM task using a 3-sec delay and a set of 16 odors, two manipulations were introduced to examine task performance under greater memory demands and at greater levels of inter-item interference. To assess the persistence of odor memory, the delay was increased to either $30 \mathrm{sec}$ or $60 \mathrm{sec}$. To examine the effect of increased inter-item interference, the size of the set of odors was reduced from 16 odors to $\mathbf{8}$ odors, so that each odor reappeared twice as frequently. Delay and interference parameters were counterbalanced such that one session was run at each combination of delay and interference; the percentage of correct responses for the entire session was used to evaluate performance at that combination of parameters. To ensure stable baseline performance, each session was followed by a return to the original parameters of 16 odors and a 3-sec delay.

\section{PROCEDURE: MORRIS WATER MAZE}

Rats were trained to locate a camouflaged escape platform in the water maze (i.e., place learning). Sessions consisted of three trials per day using a 60-sec ITI for 8 consecutive days. On each training trial, an animal was released into the maze from one of four equally spaced starting positions around the perimeter of the pool. The starting position varied from trial to trial, thus eliminating the use of a simple response strategy. The perimeter of the pool was enclosed by white canvas curtains affixed with black geometric shapes serving as discriminative extramaze cues. If an animal did not locate the escape platform within $90 \mathrm{sec}$, the ex- perimenter placed the animal on the platform, where it remained for $30 \mathrm{sec}$. Every sixth trial consisted of a probe trial to assess the development of spatial bias in the maze. During these trials, the animals swam with the platform retracted to the bottom of the pool for $30 \mathrm{sec}$, at which time the platform was raised to its normal position for completion of an escape trial. At the completion of the protocol using the hidden platform, subjects were then assessed for cue learning using a black visible platform that rose $2 \mathrm{~cm}$ above the water surface and the same testing procedures as those used in spatial training. The location of this platform varied from trial to trial in a single session of six training trials.

Escape latency was recorded and analyzed for training trials in both the place and cue learning tasks. In addition, a learning index was constructed from the probe trials during place training to reflect the animal's accuracy in searching for the escape platform. This index was based on the rat's average distance from the platform location calculated by sampling the animal's position in the maze 10 times per second during the search. Learning in the task is manifest on probe trials by a search pattern in close proximity to the escape platform location. As described in detail elsewhere (Gallagher et al. 1993), the average distances for probe trials 2-4 were weighted and summed to reflect acquisition of spatial learning over the course of training. The first probe trial was not used in the calculation of the index, because at this point in training there is routinely no age difference in probe trial performance. This continuous proximity measure provides a more efficient method of analysis than multiple measures of latency, path length, quadrants time, and annulus crossing traditionally applied to characterize probe test performance, and it has been shown to be highly sensitive to age-related spatial learning impairment (Gallagher et al. 1993).

Testing on both tasks was accomplished over a 4- to 6-week period. Data were collected in seven replications. The order of testing in the two tasks was varied among these replications. Preliminary data analysis did not indicate any effect of replication or task order. The results are, therefore, reported for all replications combined.

\section{DATA ANALYSIS}

Analysis of variance was used to examine age differences in acquisition of the cDNM task (trials

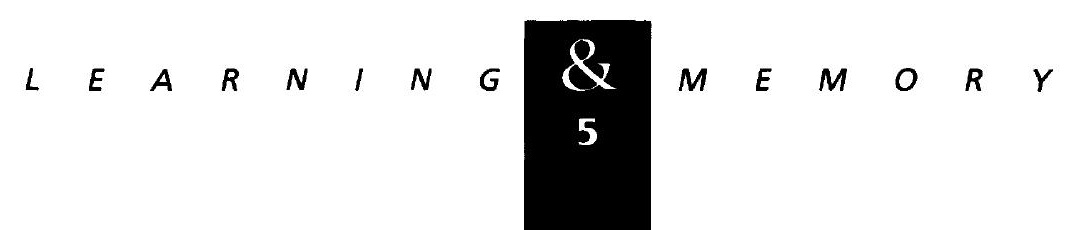


to criterion) and in performance of the task under varying delays and levels of interference [separate two-way analyses of variance (ANOVAs) for 16 and 8 odor tests with Delay and Odor Set size as within-subject factors]. Age differences were also evaluated using escape performance during place and cue training and the spatial learning index to reflect probe trial performance in the maze task.

Two types of analyses were conducted to evaluate the relationship between performances in the two tasks. For the purpose of correlational analyses, three measures from the maze task and eight measures from the cDNM task were selected. In the cDNM task, trials to reach each criterion and average performance at each delay interval during testing with the 16 and 8 odor sets (i.e., under low and high interference) were used. The spatial learning index and escape latencies at the end of place training and during the block of cue training were used to reflect performance in the water maze. Correlations within each task and intercorrelations between tasks were examined. Additional analyses examining the performance of the aged rats on the CDNM task were conducted using performance in the water maze as a reference for age-related impairment. For this purpose those aged rats that fell within the range of young performance on the spatial learning index were considered unimpaired, and those that exceeded this range were grouped as impaired in spatial learning. ANOVAs were conducted on the data obtained on CDNM performance between groups of rats who were unimpaired or impaired on the water maze.

\section{Results}

\section{CDNM TASK}

All young rats attained the level of performance required to meet both the first and second acquisition criterion. In contrast, 7 of the 24 aged rats failed to reach the initial acquisition criterion on the cDNM task and were not tested on this task further. The performance of young and successfully trained aged rats during acquisition of the cDNM task is shown in Figure 1A. Even those aged rats that did succeed were significantly impaired at reaching the initial acquisition criterion of 18 correct responses out of 20 consecutive trials on the CDNM task $[F(1,39)=5.95, P<0.01$; Fig. $1 \mathrm{~A}$, left $]$
Although this aged group required more trials to reach the additional acquisition criterion of two consecutive sessions with performance at $85 \%$ or better (Fig. 1A, right), this difference was not statistically reliable $[F(1,39)=2.68, P>0.10]$.

Following reaching acquisition criterion performance, rats were tested at different delays and levels of interference. As illustrated in Figure 2A, the aged rats performed as well as younger rats under these postacquisition testing conditions. When the 16-odor set was used (Fig. 2A, left), a repeated measures ANOVA revealed no significant difference between the groups. The performance of both groups was affected by increasing the delay, as revealed by a significant main effect of Delay $[F(1,2)=107.38, P<0.0001]$. There was no significant interaction between Age and delay. The statistical analysis of performance with the 8-odor set (Fig. 2A, right) yielded similar findings. There was no reliable effect of Age or interaction of Age with Delay. Overall performance was significantly affected by increasing the delay $[F(1,2)=33.12$, $P<0.0001$ for main effect of Delay].

\section{WATER MAZE TASK}

The assessments of spatial learning revealed an age-related deficit in both acquisition and probe trial performance. As shown in the top panel of Figure 3, the aged group was impaired in escape performance relative to the young group over the course of place training $[F(1,72)=16.52$, $P<0.0001]$. The bottom panel of Figure 3 shows the distribution of spatial learning index scores computed from probe trials for both young and aged rats. Statistical analysis of these data revealed a significant effect of Age $[F(1,39)=14.3$ $P<0.0005]$. Aged rats with spatial learning index scores falling outside the range of young animal's performance were classed as "spatial impaired" and aged rats with index scores within the range of young performance were classed as "spatial unimpaired." In contrast with these results, analysis of cue training performance revealed that aged rats were capable of proficient escape when a visible platform was provided. The average escape latencies for the young and aged groups were not significantly different on cued escape $([F(1,39)=1.50$, $P>0.10$ ]; means \pm S.E.M. were $8.58 \pm 1.13$ and $7.61 \pm 0.66$ for the young and aged groups, respectively).

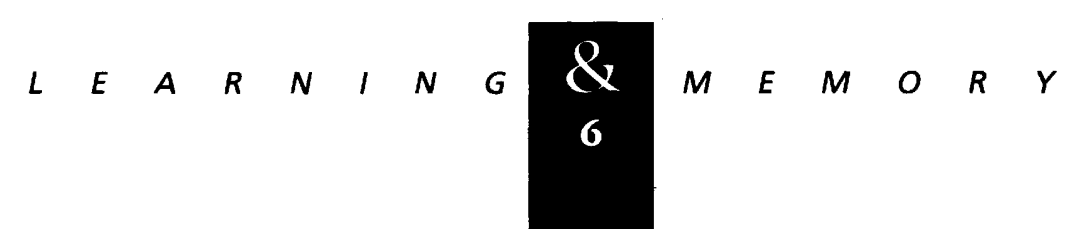


First Criterion

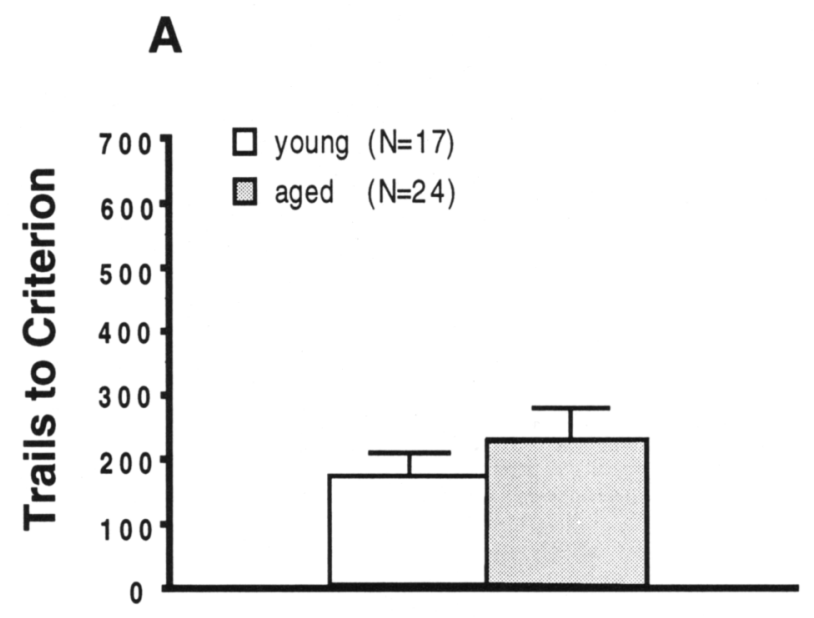

\section{Second Criterion}
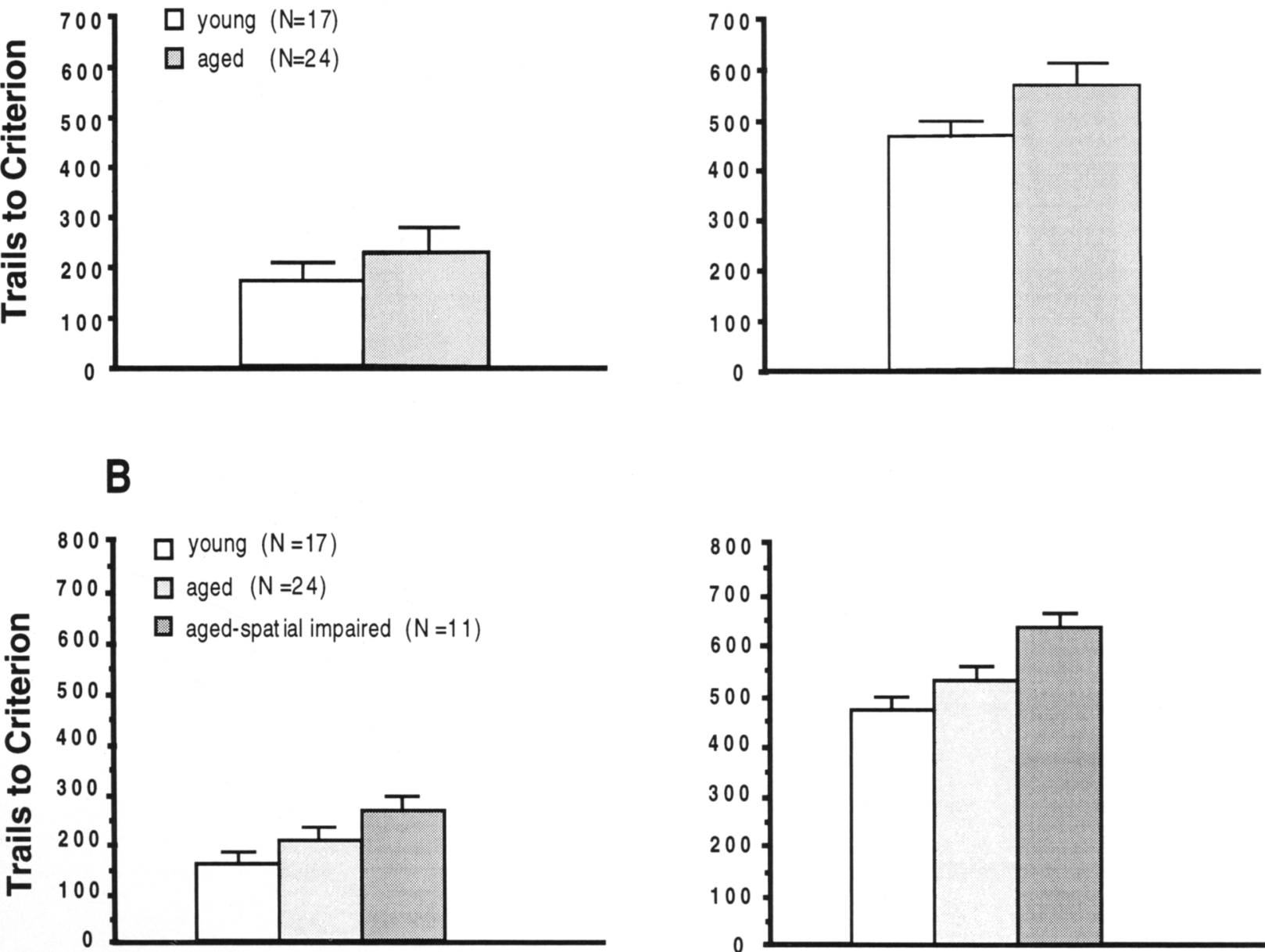

Figure 1: Performance of young and aged rats on acquisition of the cDNM task. (A) Comparison of young vs. all aged rats who succeeded in acquiring the task. (Left) Average number of trials required during acquisition to reach the intial performance criterion of 18 correct responses out of 20 consecutive trials. (Right) Average number of trials required to reach an additional criterion of two consecutive sessions with performance of $85 \%$ or better. (B) Comparison of young vs. aged rats with the latter divided into subgroups that were impaired or unimpaired in the Morris water maze task. (Left and right) Illustrations of performance for the same acquisition criteria as those in panels above.

RELATIONSHIPS BETWEEN DIFFERENT ASPECTS OF PERFORMANCE ON CDNM AND SPATIAL WATER MAZE TASKS AND BETWEEN PERFORMANCE ACROSS TASKS

Correlations among the measures obtained in CDNM were generally higher for aged rats than for young rats (Table 1). For example the correlation $(r)$ between trials to reach the first and second criteria was $0.67(P<0.001)$ for aged rats but only 0.03 for young. Performance at each delay under high interference (8-odor-set) was reliably correlated with trials to each acquisition criterion for the aged rats $(P<0.01)$, but no reliable correlations were obtained in the comparable analyses for young rats. Correlations among the measures obtained from water maze performance were similar to those previously reported (Table 2; Gallagher et al. 1993). Little relationship was evident between cued training performance and measures in the place learning task for either age group ( $r$ values ranging from -0.18 to 0.25 ). The spatial learning

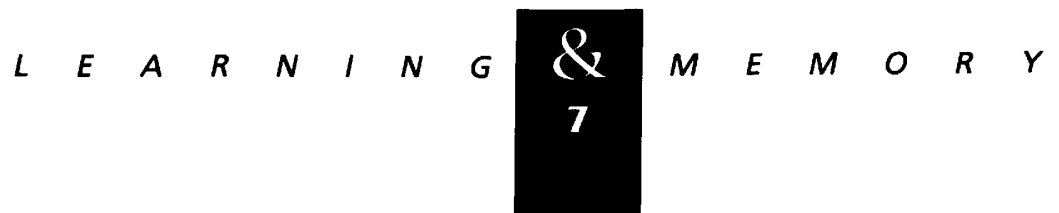




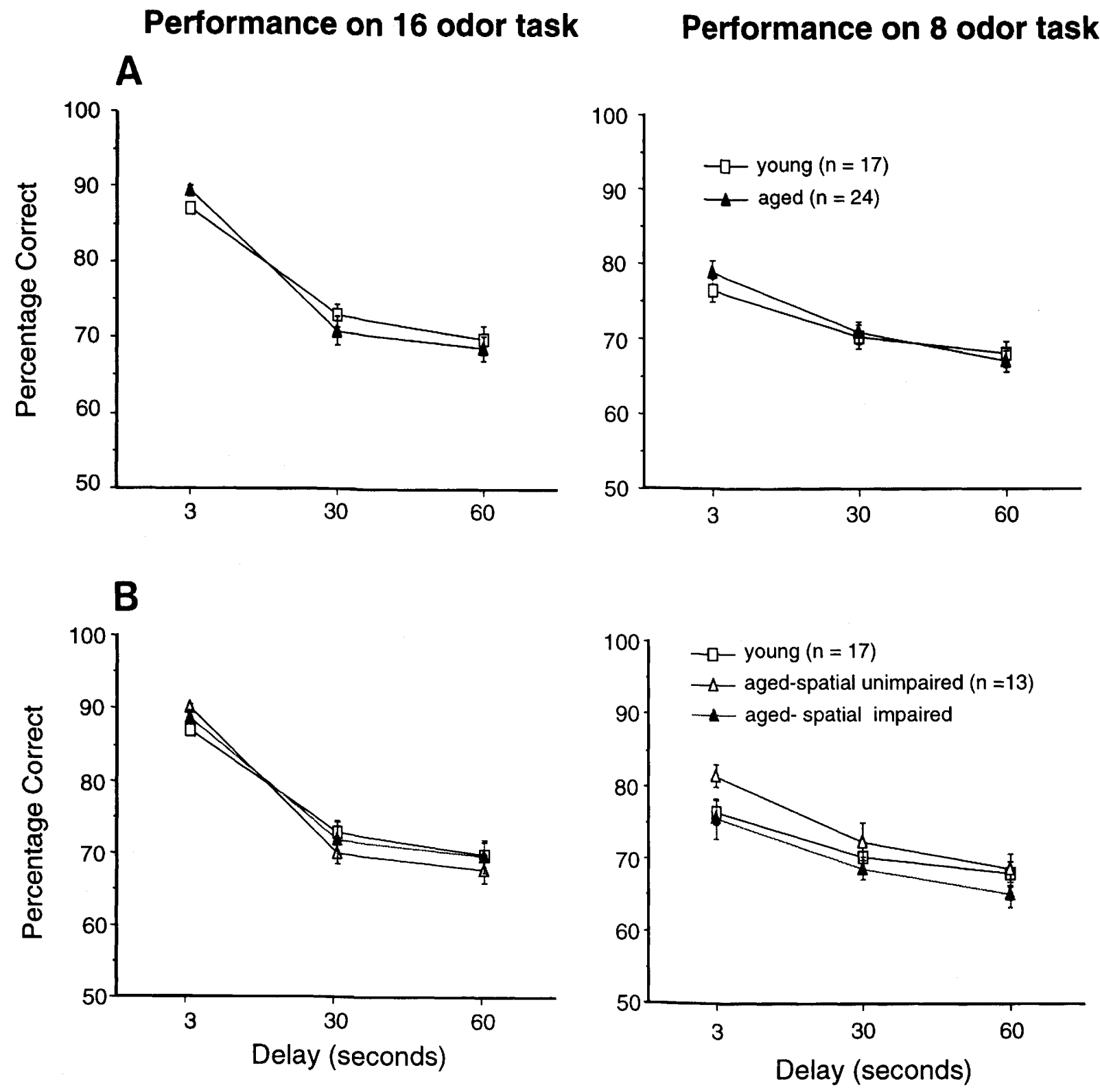

Figure 2: Performance of young and aged rats across varying memory delays and interference. $(A)$ Comparison of young vs. all aged rats. (Left) Odor set $=16$; (right) Odor set $=8$. (B) Comparison of young vs. aged rats with the latter divided into subgroups that were impaired or unimpaired in the Morris water maze task. (Left and right) Illustrations of performance for the same size odor sets as those in panels above.

index during probe trial performance was related to escape latency during training, although this was only a modest correlation $(r=0.46, P<0.02$ for aged; $r=0.36, P>0.05$ for young).

Comparing performance across tasks, in a few cases the correlation coefficients for measures on the two tasks were moderately strong ( $r$ values ranging from -0.33 to 0.43 ; see Table 3 ). However, none of these correlations were reliable for either age group. Nevertheless, in contrast to the subject intercorrelations, our comparisons of cDNM performance based on grouping spatial-impaired and spatial-unimpaired subjects revealed significant associations between age-related impairments on the two tasks. The performance of the young rats and the two subgroups of aged rats (spatial unimpaired and spatial impaired) during acquisition of the cDNM task is shown in Figure

$$
\begin{array}{llllllll|c|ccccccc}
L & E & A & R & N & I & N & G & \mathbf{Q} & M & E & M & O & R & Y \\
\mathbf{8} & & & & & &
\end{array}
$$


Training Trial Average Latency

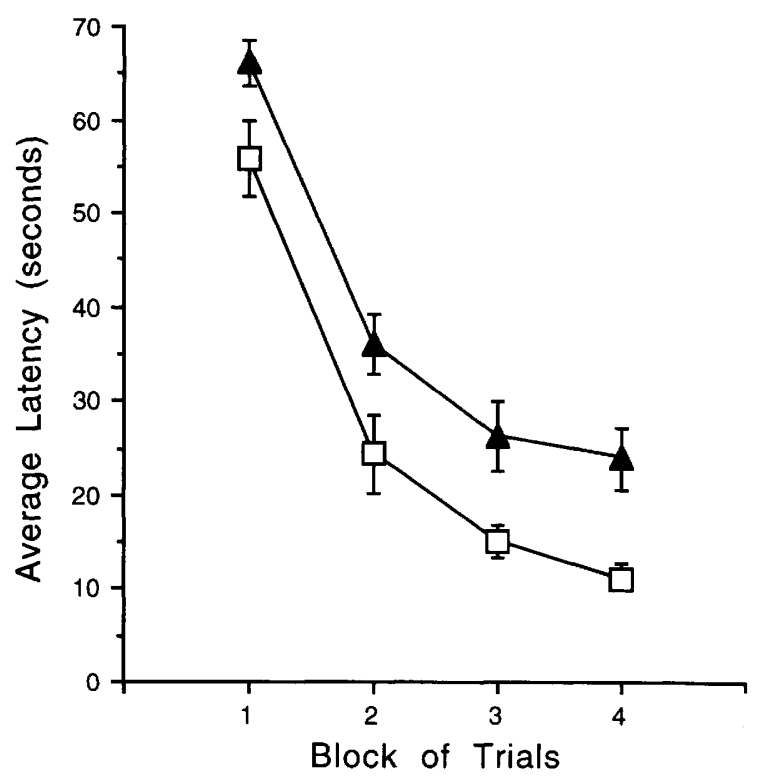

Spatial Learning Index Distribution

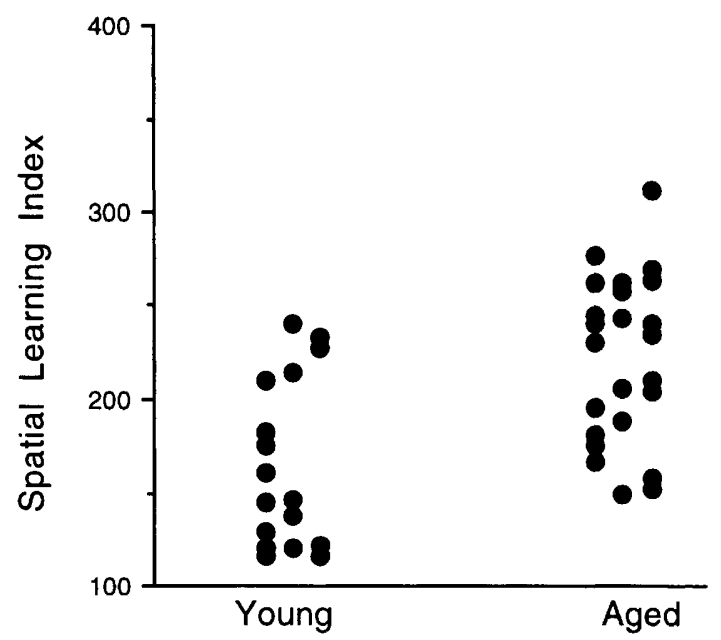

Figure 3: Performance of young $(\square)$ and aged $(\boldsymbol{\Delta})$ rats on the Morris water maze task. (Top) Mean escape latency on successive blocks of training trials; (bottom) distribution of the spatial learning index based on probe trial performance. Subgroups of aged rats in Figs. $1 \mathrm{~B}$ and $2 \mathrm{~B}$ are based on the learning index scores shown at bottom here. Spatial unimpaired aged rats had scores within the range of the young group; the remaining aged rats were designated spatial impaired.

1B. Subgrouping the aged animals on the basis of spatial learning in the water maze revealed that aged rats impaired in that task were also signifi- cantly impaired at learning the initial acquisition criterion of 18 correct responses out of 20 consecutive trials on the cDNM task $[F(2,38)=5.07$, $P<0.01$; Fig. 1B, left]. Post hoc comparisons (Neuman-Keuls) revealed that performance of the young and spatial-unimpaired aged rats did not differ, but both of these groups differed reliably from the spatial-impaired aged rats. Although a similar analysis on acquisition of the additional acquisition criterion of two consecutive sessions with performance at $85 \%$ or better revealed a similar trend (Fig. 1B, right), these group differences did not reach statistical significance $[F(2,38)=2.62$, $P<0.08$; graph on the right].

Performance at different delays and levels of interference for the cDNM task was also examined when aged rats were subgrouped by their spatial learning ability (Fig. 2B). Analyses of these data failed to reveal any differences among the groups. In both the 16- and 8-odor set testing conditions, all groups were similarly affected by increasing the delay $([F(2,2)=112.3, P<0.0001]$ and $[F(2,2)$ $=35.67, P<0.0001]$ for main effects of Delay with the 16- and 8-odor sets, respectively). No main effects of Age or interactions of Age with Delay were obtained in these analyses.

Data from seven aged rats were excluded from all final analyses because these subjects failed to reach either the first or second criteria on the cDNM task. Four failed at the first acquisition criterion, and the remaining three failed to reach the second acquisition criterion. All of these animals completed testing in the water maze, where performance was not systematically related to an inability to acquire the cDNM task. Five of these aged rats had spatial learning index scores that fell within the range of young rats, whereas the remaining two were clearly impaired in the water maze task. Analysis of cued water maze performance revealed that these aged rats were capable of proficient escape when a visible platform was provided. The average escape latencies for these seven aged animals and those aged animals successfully completing cDNM was not different (means \pm SEM S.E.M. were $11.97 \pm 2.46$ and $7.61 \pm 0.66$, respectively). Moreover, no discernible differences in water maze learning were apparent on the basis of whether these animals failed to reach either the first or second acquisition criterion in the cDNM task. Thus, the rats who were most impaired in acquiring the cDNM task were not especially impaired in the water maze, suggesting there may be different age-related cognitive

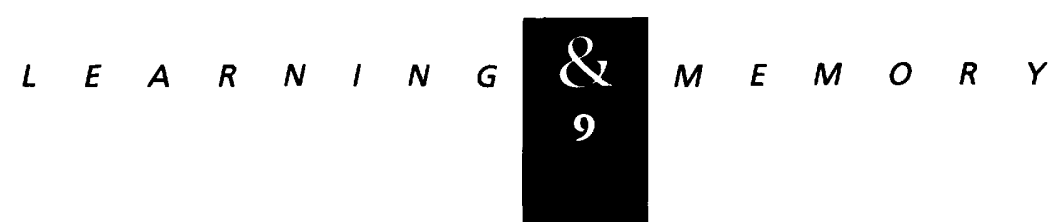


Table 1: Correlations within the odor task

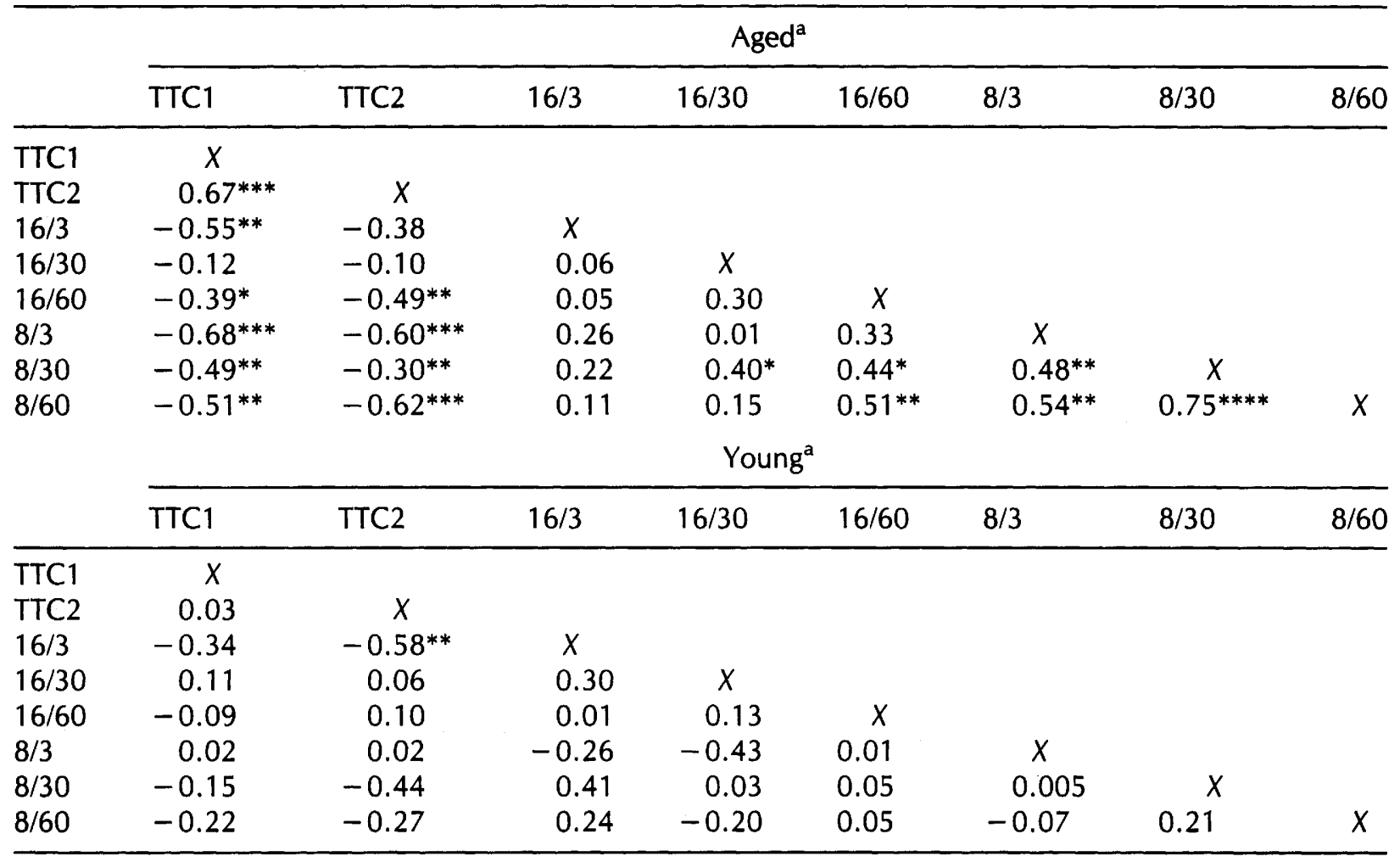

TTC1 indicates trials to first acquisition criterion. TTC2 indicates trials to second acquisition criterion. Measures shown as two numbers indicate percent correct with various sizes of the odor stimulus set (first number) and various memory delays (second number).

${ }^{a} P<0.05 ;{ }^{* *} P<0.01 ;{ }^{* * *} P<.001 ;{ }^{* * *} P<0.0001$

deficiencies leading to poor recognition memory performance.

\section{Discussion}

There were two principle findings of this research. First, aged rats were impaired in the early stage on acquisition of an odor-guided cDNM task. Moreover, a significant subset of aged rats could not acquire the task even with substantially more training that all young rats. Nevertheless, aged rats who succeeded in learning the CDNM task showed no deficit in recognition memory under conditions of increased delay and interference. Second, a subgroup of rats who were impaired on a separate test of spatial learning in the Morris water maze performed especially poorly on CDNM acquisition. Despite these group associations, there
Table 2: Correlations within the spatial task

\begin{tabular}{|c|c|c|c|}
\hline & \multicolumn{3}{|c|}{ Aged } \\
\hline & $\begin{array}{l}\text { learning } \\
\text { index }^{a}\end{array}$ & $\begin{array}{l}\text { spatial } \\
\text { latency }\end{array}$ & $\begin{array}{l}\text { cue } \\
\text { latency }\end{array}$ \\
\hline Learning index & $X$ & & \\
\hline Spatial latency & $0.46^{*}$ & $X$ & \\
\hline \multirow[t]{3}{*}{ Cue latency } & 0.18 & 0.25 & $x$ \\
\hline & \multicolumn{3}{|c|}{ Young } \\
\hline & $\begin{array}{l}\text { learning } \\
\text { index }\end{array}$ & $\begin{array}{l}\text { spatial } \\
\text { latency }\end{array}$ & $\begin{array}{l}\text { cue } \\
\text { latency }\end{array}$ \\
\hline Learning index & $x$ & & \\
\hline Spatial latency & 0.36 & $X$ & \\
\hline Cue latency & -0.18 & 0.018 & $X$ \\
\hline
\end{tabular}

${ }^{a *} P<0.05$.

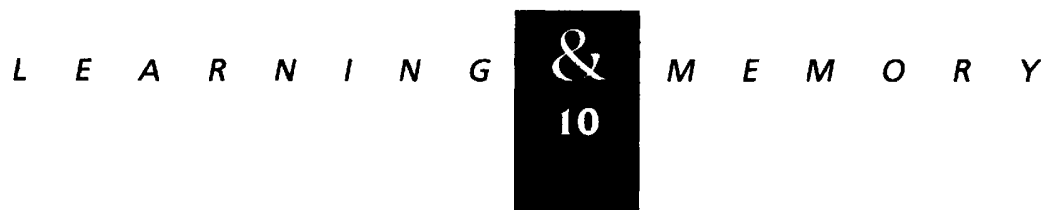


Table 3: Intercorrelations of performance between CDNM and water maze

\begin{tabular}{lccccc}
\hline \multicolumn{5}{c}{} & \multicolumn{5}{c}{ Aged } \\
\cline { 2 - 6 } & index 30-3 & cue latency & latency 1-4 & $\begin{array}{c}\text { block } 4 \\
\text { latency }\end{array}$ & $\begin{array}{l}\text { block } 4 \\
\text { index }\end{array}$ \\
\hline TTC1 & 0.23 & -0.14 & 0.04 & 0.25 & 0.14 \\
TTC2 & 0.27 & -0.19 & 0.04 & 0.35 & 0.13 \\
16 odors (\%) & 0.19 & 0.43 & 0.12 & 0.24 & 0.35 \\
8 odors (\%) & -0.33 & 0.03 & -0.03 & -0.23 & -0.23 \\
& & & Young & & \\
\cline { 2 - 6 } & & & & block 4 & block 4 \\
& index 30-3 & cue latency & latency 1-4 & latency & index \\
\hline TTC1 & 0.02 & 0.05 & 0.08 & -0.19 & -0.11 \\
TTC2 & -0.06 & 0.23 & 0.07 & 0.07 & -0.03 \\
16 odors (\%) & 0.18 & -0.06 & 0.08 & 0.06 & 0.18 \\
8 odors (\%) & 0.08 & -0.06 & 0.004 & 0.17 & 0.09 \\
\hline
\end{tabular}

TTC1 indicates trials to first acquisition criterion. TTC2 indicates trials to second acquisition criterion. Other measures indicate percent correct with 16- or 8-odor stimulus sets.

was no significant within-subject correlation between performance on CDNM and spatial learning. Each of these results will be discussed in turn, focusing on considerations of the nature of the aged-related cognitive deficits and of the brain structures compromised in aging.

\section{AGE-RELATED SELECTIVE IMPAIRMENT ON CDNM ACQUISTION}

Several previous studies have examined the performance of aged rats in a variety of recognition memory and delayed matching- and nonmatching-to-sample tasks; the results of these studies are variable both with regard to acquisition and delay performance. The dissociation between impaired acquisition and subsequently intact performance with increasing delays and interference in the present study extends these findings and may be helpful for interpreting the equivocal findings from these other studies. Most similar to our results are those of Aggleton et al. (1989) indicating that 16- to 20-month-old rats were impaired on acquisition but not delay performance of a delayed nonmatch-to-sample task where the memory cues consisted of goal boxes that appeared only once as the to-be-remembered memory cue. In that study aged rats were also impaired in acquisition but not delay performance of a delayed nonmatch-to-sam- ple task where the cues were one of two turns in a T-maze. In addition, Cavoy and Delacour (1993) found that 18- and 24-month-old rats demonstrated preserved delayed retention, even at rather long ( $300 \mathrm{sec}$ ) intervals, in task where reduced exploration during representation of a previously novel object was used as a measure of recognition.

In contrast to the observation of preserved delay performance in each of these studies, others have reported delay-related deficits in aged rats. For example, in the Cavoy and Delacour (1993) study, in contrast to the sparing of delayed object recognition, the same aged subjects were severely impaired in delayed $(90 \mathrm{sec})$ recognition of an arm in a spatial maze. Also, Dunnett et al. (1988) reported that 15- and 24-month-old rats had a delay-dependent deficit in matching and nonmatching to sample in a task where rats had to remember which of two retractable levers was presented: In this study aged animals were not impaired during initial acquisition of the task when no delay was imposed between the sample and match stimulus presentations. Finally, Winocur (1992) found that 26-month-old rats were impaired in both acquisition and delay performance in two matching tasks, one that involved matching the identity of choice stimuli to one of two light-intensity sample cues that were used on every trial and another that involved matching a left or right lever bar press

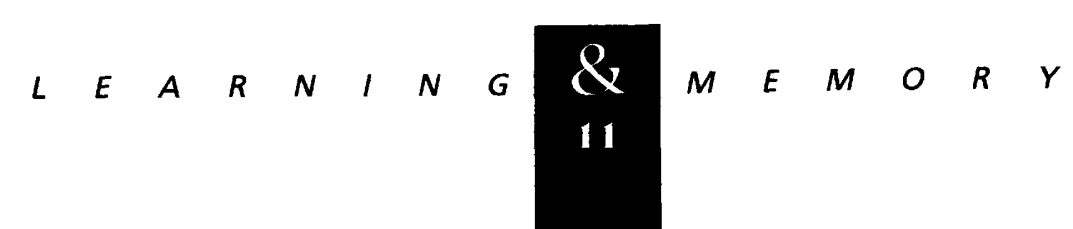


choice response to a left or right light sample stimulus presented near the corresponding levers.

Multiple factors, including strain and numerous procedural differences between these studies, could be the basis for the divergent results across these studies. One possibility is that different outcomes can be attributed to differences in the nature of the memory cues and frequency with which they are presented across these studies. Thus, age-related acquisition deficits were observed in all tasks, except the Dunnett et al. (1988) study where no delay was used during training. Even in that study, an acquisition deficit was observed across delays as soon as a 0 - to 24sec random delay was introduced. Postacquisition delay-dependent performance deficits were observed in tasks that involved complex memory cues defined by spatial, temporal, or conditional relations among multiple stimuli. Thus, in the Cavoy and Delacour (1993) study, a delay performance deficit was observed in memory for the relative location of one of two maze arms. Similarly, deficits were observed in tasks that demanded memory for the relative recency ("working memory") of one of two levers (Dunnett et al. 1988), light intensities (Winocur 1991) or conditional cue locations (Winocur 1992). In contrast, delay-dependent postacquisition performance was not impaired in tasks that employed nonrepeating (Aggleton et al. 1989; Cavoy and Delacour 1993) or seldom-repeating (this study) discrete stimuli. The only exception to this characterization of the combined results is the absence of a delay-dependent deficit for spatial cues in the Aggleton et al. (1989) study. However, even in that study they did find a deficit for spatial cues when the number of to-be-remembered items was increased from one to two.

The pattern of findings on delayed retention performance in studies on aging rats parallels an accounting of memory deficits consequent to hippocampal damage in both rats and monkeys (Cohen and Eichenbaum 1993; for review, see Eichenbaum et al. 1992, 1994). Thus, as observed in aged rats, experimental damage limited to the hippocampus results in impairments on delayed retention when the task requires remembering spatial or conditional relations among stimuli or working memory for the relative recency of repeating cues but not when the task requires memory for discrete, nonrepeating or seldom repeating stimuli. In contrast, damage to the perirhinal and entorhinal cortical areas surrounding the hippocampus produces a pattern of performance opposite to that observed in aged rats, that is, sparing of acquisition combined with impairment in delayed retention of discrete stimuli (Otto and Eichenbaum 1992). These considerations suggest that the cortex surrounding the hippocampus is not substantially compromised by aging in rats; any age-related dysfunction of the hippocampus itself would not be expected to be reflected within a deficit in cDNM performance.

Regarding the observed deficit in CDNM acquisition, several findings in the present research point to the possible involvement of prefrontal cortex dysfunction in the aged rat. Damage to the prefrontal cortex, in both monkeys (GoldmanRakic 1990) and rats (Otto and Eichenbaum 1992), results in acquisition deficits on delayed nonmatch to sample. Furthermore, in the latter study, no impairment was observed on CDNM delay performance following prefrontal lesions as long as the stimulus set was large. A delay-dependent deficit was observed when the stimulus set was reduced, suggesting that prefrontal lesions may result in increased susceptibility to interference. In the present study, the absence of sensitivity to increased interference in aged rats may have been because of the relatively mild acquisition deficit compared to that following explicit damage to the prefrontal area. Thus, the present results are most consistent with a selective dysfunction of prefrontal cortex and not the hippocampal system.

Winocur (1991, 1992) also concluded that prefrontal dysfunction might be responsible for age-related acquisition deficits in some behavioral paradigms. In his studies, rats with prefrontal damage were selectively impaired in the acquisition phase of delayed and conditional matching tasks, and aged rats showed a milder version of this impairment. In contrast, rats with hippocampal lesions acquired these tasks normally but were selectively impaired as the memory delay was increased. Unlike the present results, the performance of aged rats deteriorated with increased delays, leading Winocur $(1991,1992)$ to conclude that both prefrontal and hippocampal function may be compromised in aging. However, as pointed out above, unlike the present research and the Aggleton et al. (1989) study, Winocur's tasks employed memory cues that relied on the processing of temporal or conditional relations among stimuli. The present findings suggest that the delay-dependent component of the perfor-

$$
\text { ………….... }
$$


mance deficit in Winocur's and other studies is attributable to the demand for relational processing of the memory cues.

\section{SUBGROUP ASSOCIATION, BUT NOT WITHIN-SUBJECT CORRELATION, BETWEEN DEFICITS IN CDNM AND SPATIAL LEARNING PERFORMANCE}

Aged rats were impaired in place learning in the Morris water maze. Furthermore, the dissociation of age-related impairment in spatial learning and sparing of cued training found in this experiment is similar to that reported in many other studies (Gage et al. 1984a,b; Gallagher and Pellymounter 1988; Gallagher and Burwell 1989; Issa et al. 1990; Gallagher et al. 1993). Moreover, as found in each of those studies, only a subset of the aged rats performed outside the range of scores of young adults in spatial learning. When aged rats were separated into subgroups according to their spatial learning performance, only the spatial-impaired subgroup were significantly impaired on acquisition of the cDNM task. However, despite this subgroup association between spatial and CDNM impairments, there was no within-subject correlation of performance on the two tasks in aged or young rats. Furthermore, those aged rats who could not acquire the CDNM task were not the most impaired at spatial learning. This pattern of findings suggests the influence of multiple age-related factors in the deficits observed, with some factor or factors associated with deficits in both tasks and another factor or factors involved in each task independently. A consideration of the neuropsychological bases of performance in the water maze and in the cDNM tasks may shed light on both task-general and task-specific factors.

As noted in the Introduction, lesions of the hippocampus proper in young rats have different effects on spatial learning versus recognition memory performance in the cDNM task. Hippocampal lesions produce deficits in Morris water maze performance (Gallagher and Holland 1989) but not in cDNM acquisition or retention across delays (Otto and Eichenbaum 1992; Wilner et al. 1993). In this way, the performance of aged rats in this study parallels the findings on young rats with lesions of hippocampus. This suggests the possibility that effects of aging present in the hippocampus might account for the age-related deficits we observed in the water maze independent of performance on the cDNM task. There is considerable evidence that the medial temporal area is among the first areas to show significant anatomical and physiological deterioration with normal aging (Albert and Moss 1988; Killiany et al. 1993). Such susceptibility might account for the fact that cognitive functions associated with this structure are particularly sensitive to the effects of aging. In contrast to the effects of damage to the hippocampus, lesions of perirhinal and entorhinal cortices in young rats produce delay-dependent deficits in the recognition memory task employed in the present study (Otto and Eichenbaum 1992). Because delay performance of the aged rats was remarkably preserved in the cDNM task, substantial effects of aging in these cortical areas may not be present in the rat or may occur at a later stage in the aging process. Conversely, as described above, age-related changes in the prefrontal area could account for deficits in CDNM acquisition. In particular, selective orbital prefrontal cortex lesions in young rats produce selective deficits on olfactory and not spatial learning (Eichenbaum et al. 1983). Thus, selective dysfunction of the orbital prefrontal area could be responsible for the specific deficit in olfactory learning unrelated to a deficit on spatial performance.

One possibility for the anatomical locus of an age-related factor in common between the two tasks is a general compromise of prefrontal cortical function. Thus, in the Eichenbaum et al. (1983) study, a double dissociation indicating a critical role for the medial prefrontal area in spatial memory and for the orbital prefrontal area in olfactory learning was observed. Combined with other studies showing that medial prefrontal damage results in mild deficits in Morris water maze performance (Kolb et al. 1983; Kolb 1990) and in acquisition of delayed nonmatching to sample in monkeys (Kowalska et al. 1991), it is possible that a pervasive age-related deterioration of the prefrontal cortex would result in an impairment of performance on both tasks. The prefrontal cortex might play an important and general role in acquiring procedural knowledge, and, within that general role, specific regions of prefrontal cortex might be critical to acquiring the rules that govern performance in particular tasks. A similar position has been forwarded by Winocur and Moscovitch (1990) and Winocur (1992) who reported that lesions to the medial prefrontal cortex led to a selective impairment in acquiring skill-related information specific to solving a maze task. These

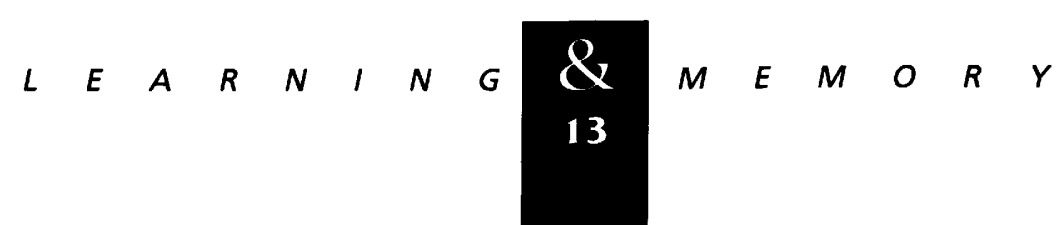


considerations suggest that a common factor in age-related cognitive capacity might be dysfunction of the prefrontal cortex, suggesting that prefrontal dysfunction could account for the full pattern of impairment and preserved capacities and the subgroup associations observed in the present study.

\section{CONCLUSIONS}

The present results are in general agreement with theoretical positions advancing functional subdivisions of memory components in the hippocampal system and prefrontal cortex. The selectivity of impairment observed in each task suggests that particular memory systems or parts of these systems are disproportionately compromised in aged organisms. Also, the present data add to increasing evidence of both prefrontal and hippocampal system dysfunction associated with normal aging. Furthermore, the broad scope of the deficit across tasks, combined with the observed relationship between the selective impairments between subgroups, suggests that multiple brain systems deteriorate together in aging. Finally and more broadly, the neuropsychological approach appears to be a fruitful one for studies of cognitive decline with age in rodents. The challenge for this strategy is one of describing the fundamental cognitive attributes that are common to tasks on which the performance of aged subjects is impaired or preserved and of identifying the cellular and molecular bases that mediate performance on those tasks.

\section{Acknowledgments}

This work was supported by National Institute on Aging grant P01 AG09973.

The publication costs of this article were defrayed in part by payment of page charges. This article must therefore be hereby marked "advertisement" in accordance with 18 USC section 1734 solely to indicate this fact.

\section{References}

Aggleton, J.P., H.S. Blindt, and J.M. Candy. 1989. Working memory in aged rats. Behav. Neurosci. 103: 975-983.

Albert, M.S. and M.B. Moss. 1988. Geriatric Neuropsychology. Guilford Press, New York.

Barnes, C.A. 1979. Memory deficits associated with senescence: A neurophysiological and behavioral study in the rat. J. Comp. Physiol. Psychol. 93: 74-104.
Cavoy, A. and J. Delacour. 1993. Spatial but not object recognition is impaired by aging in rats. Physiol. Behav. 53: $527-530$

Cohen, N.J. and H. Eichenbaum. 1993. Memory, amnesia, and the hippocampal system. MIT Press, Cambridge, MA.

Collier, T.J. and P.D. Coleman. 1991. Divergence of biological and chronological aging: Evidence from rodent studies, Neurobiol. Aging 12: 685-693.

de Leon, M.J., G. Smith, A.E. George, T. McRae, J. Golomb, A. Convit, A. Kluger, W. Tsui, S.H. Ferris, and A.P. Wolf. 1992. CT, MRI, and PET studies of hippocampal pathology in Alzheimer's disease. In Neurodevelopment, aging and cognition (ed. I. Kostovic, S. Knezevic, H.M. Wisniewski, and G.J. Spilich), pp. 323-335. Birkhauser Press, Boston, MA.

Dunnett, S.B., J.L. Evenden, and S.D. Iverson. 1988. Delay-dependent short-term memory deficits in aged rats. Psychopharmacology 96: 174-180.

Eichenbaum, H., R.A. Clegg, and A. Feeley. 1983. Reexamination of functional subdivisions of the rodent prefrontal cortex. Exp. Neurol. 79: 434-451.

Eichenbaum, H., T. Otto, and N.J. Cohen. 1992. The hippocampus-What does it do? Behav. Neural Biol. 57: $2-36$

1994. Two functional components of the hippocampal memory system. Brain Behav. Sci. 17: 449-518.

Gaffan, D. 1974. Recognition impaired and association intact in the memory of monkeys after transection of the fornix. $J$. Comp. Physiol. Psychol. 86: 1100-1109.

Gage, F.H, P.A.T. Kelly, and A. Bjorklund. 1984a. Regional changes in brain glucose metabolism reflect cognitive impairments in aged rats. J. Neurosci. 4: 2856-2865.

Gage, F.H, S.B. Dunnett, and A. Bjorklund. 1984b. Spatial learning and motor deficits in aged rats. Neurobiol. Aging 5: $43-48$.

Gallagher, M. and R.D. Burwell. 1989. Relationship of age-related decline across several behavioral domains. Neurobiol. Aging 10: 691-708.

Gallagher, M. and P. Holland. 1989. Preserved configural learning and spatial learning impairment in rats with hippocampal damage. Hippocampus 2: 81-88.

Gallagher, M. and M.A. Pellymounter. 1988. Spatial learning deficits in old rats: A model for memory decline in the aged. Neurobiol. Aging 9: 549-556.

Gallagher, M., R.D. Burwell, and M. Burchinal. 1993. Severity of spatial learning impairment in aging: Development of a learning index for performance in the morris water maze. Behav. Neurosci. 107: 618-626. 
Gallagher, M., A.H. Nagahara, and R.D. Burwell. 1995. Cognition and hippocampal systems in aging: Animal models. In Brain and memory: Modulation and mediation of neuroplasticity (ed. J.L. McGaugh, N. Weinberger, and G. Lynch), Oxford University Press, New York (In press.)

Goldman-Rakic, P. 1990. Cortical localization of working memory. In Brain organization and memory: Cells, systems, and circuits (ed. J.L. McGaugh, N.M. Weinberger, and G. Lynch), pp. 285-298. Oxford University Press, New York.

Golomb, J., A. Kluger, M.J. de Leon, A.E. George, C. Tarshish, and S.H. Ferris. 1993. Hippocampal atrophy in normal aging: An association with recent memory impairment. Arch. Neurol. 50: 967-973.

Golomb, J., A. Kluger, M.J. de Leon, S.H. Ferris, A. Convit, M.S. Mittleman, J. Cohen, H. Rusniek, S. De Santi, and A.E. George. 1994. Hippocampal formation size in normal human aging: A correlate of delayed secondary memory performance. Learn. Mem. 1: 45-54.

Issa, A.M., W. Rowe, S. Gauthier, and M.J. Meany. 1990. Hypothalamic-pituitary-adrenal activity in aged, cognitively impaired and cognitively unimpaired rats. J. Neurosci. 10: $3247-3254$.

Killiany, R.J., M.B. Moss, M.S. Albert, T. Sandor, J. Tieman, and F. Jolesz. 1993. Temporal lobe regions on magnetic resonance imaging identify patients with early Alzheimer's disease. Arch. Neurol. 50: 949-954.

Kolb, B. 1990. Prefrontal cortex. In The cerebral cortex (ed. B. Kolb and R.C. Tees), pp. 437-458. MIT Press, Cambridge, MA.

Kolb, B., R.J. Sutherland, and I.Q. Wishaw. 1983. A comparison of the contributions of the frontal and parietal association cortex to spatial localization in rats. Behav. Neurosci. 97: 13-27.

Kowalska, D.M., J. Bachevalier, and M. Mishkin. 1991. The role of the inferior prefrontal convexity in performance of delayed nonmatching-to-sample. Neuropsychologia 29: $583-600$.

Meunier, M. J. Bachevalier, M. Mishkin, and E.A. Murray. 1993 Effects on visual recognition of combined and separate ablations of the entorhinal and perirhinal cortex in rhesus monkeys. J. Neurosci. 13: 5418-5432.

Mishkin, M. 1982. A memory system in the monkey. Phil. Trans. R. Soc. London B 209: 85-95.

Mitchell, S.J., J.N.P. Rawlins, O. Steward, and D.S. Olton. 1982. Medial septal area lesions disrupt theta rhythm and alter cholinergic staining in medial entorhinal cortex and produce impaired radial arm maze behavior in rats. I. Neurosci. 2: 292-302.

Morris, R.G.M., P. Garrud, J.N.P. Rawlins, and J. O'Keefe. 1982. Place navigation impaired in rats with hippocampal lesions. Nature 297: 681-683.
Morris, R.G.M., E. Anderson, G.S. Lynch, and M. Baudry. 1986. Selective impairment of learning and blockade of long-term potentiation by an $\mathrm{N}$-methyl-D-aspartate receptor antagonist, AP5. Nature 319: 774-776.

Morris, R.G.M, F. Schenk, F. Tweedie, and L.E. Jarrard. 1990. Ibotenate lesions of hippocampus and/or subiculum: Dissociating components of allocentric spatial learning. Eur. J. Neurosci. 2: 1016-1028.

Nagahara, A., T. Otto, and M. Gallagher. 1995. Entorhinal/perirhinal lesions impair performance on two versions of place learning in the Morris water maze. Behav. Neurosci. 109: 3-9.

O'Boyle Jr., V.J., E.A. Murray, and M. Mishkin. 1993. Effects of excitotoxic amygdalo-hippocampal lesions on visual recognition in rhesus monkeys. Soc. Neurosci. Abstr. 19: 438 .

Otto, T. and H. Eichenbaum. 1992. Complementary roles of the orbital prefrontal cortex and the perirhinal-entorhinal cortices in an odor-guided delayed-nonmatching-to-sample task. Behav. Neurosci. 106: 762-775.

Rapp, P.R. and D.G. Amaral. 1989. Evidence for task-dependent memory dysfunction in the aged monkey. J. Neurosci. 9: 3568-3576.

1991. Recognition memory deficits in a subpopulation of aged monkeys resemble the effects of medial temporal lobe damage. Neurobiol. Aging 12: $481-486$.

1992. Individual differences in the behavioral and neurobiological consequences of normal aging, Trends Neurosci. 15: 340-345.

Rapp, P.R. and W.C. Heindel. 1994. Memory systems in normal and pathological aging. Curr. Opin. Neurol. 7: 294-298.

Sandor, T., F. Jolesz, J. Tieman, R. Kikinis, K. Jones, and M. Albert. 1992. Comparative analysis of computed tomographic and magnetic resonance imaging scans in alzheimer patients and controls. Arch. Neurol. 49: 381-384.

Shimamura, A.P. 1994. Neuropsychological perspectives on memory and cognitive decline in normal human aging. Sem. Neurosci. 6: 387-394.

Suzuki, W.A., S. Zola-Morgan, L.A. Squire, and D.G. Amaral. 1993. Lesions of the perirhinal and parahippocampal cortices in the monkey produce long-lasting memory impairment in the visual and tactual modalities. I. Neurosci. 13: $2430-2451$.

Wilner, J., T. Otto, M. Gallagher, and H. Eichenbaum. 1993. Hippocampal lesions that impair place learning facilitate delayed nonmatching performance in rats. Soc. Neurosci. Abstr. 19: 358.

Winocur, G. 1991. Conditional learning in aged rats: 


\section{Zyzak et al.}

Evidence of hippocampal and prefrontal cortex impairment. Neurobiol. Aging 13: 131-135.

1992. A comparison of normal old rats and young adult rats with lesions to the hippocampus or prefrontal cortex on a test of matching-to-sample. Neuropsychologia 9: 769-781.

Winocur, G. and M. Moscovitch. 1990. Hippocampal and prefrontal cortex contributions to learning and memory: Analysis of lesion and aging effects on maze learning in rats. Behav. Neurosci. 104: 544-551.

Zola-Morgan, S. and L.R. Squire. 1985. Medial temporal lesions in monkeys impair memory on a variety of tasks sensitive to human amnesia. Behav. Neurosci. 99: 22-34.

Zola-Morgan, S., L.R. Squire, and D.G. Amaral. 1989. Lesions of the hippocampal formation but not lesions of the fornix or mammillary nuclei produce long-lasting memory impairment in the monkey. J. Neurosci. 9: 898-913.

Zola-Morgan, S., L.R. Squire, D.G. Amaral, and W.A. Suzuki. 1989. Lesions of perirhinal and parahippocampal cortex that spare the amygdala and hippocampal formation produce severe memory impairment. I. Neurosci.

9: $4355-4370$.

Received November 29, 1994; accepted in revised form February 10, 1995. 


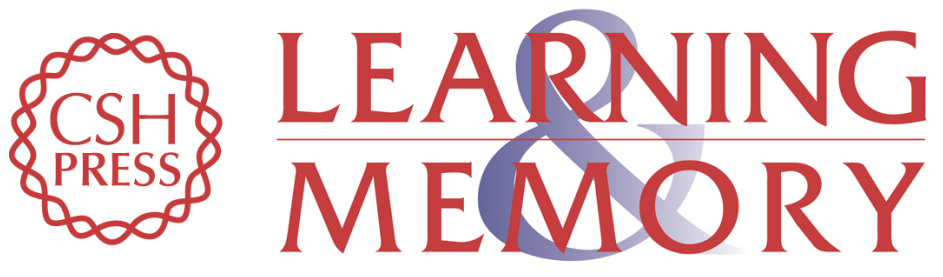

\section{Cognitive decline associated with normal aging in rats: a neuropsychological approach.}

D R Zyzak, T Otto, H Eichenbaum, et al.

Learn. Mem. 1995, 2:

Access the most recent version at doi:10.1101//m.2.1.1

References This article cites 44 articles, 9 of which can be accessed free at:

http://learnmem.cshlp.org/content/2/1/1.full.html\#ref-list-1

License

Email Alerting

Receive free email alerts when new articles cite this article - sign up in the box at the Service top right corner of the article or click here. 